\title{
A Prototype Evaluation of a Tamper-resistant High Performance Blockchain-based Transaction Log for a Distributed Database
}

\author{
(short paper)
}

\author{
L. Aniello, R. Baldoni, E. Gaetani and F. Lombardi \\ Research Center of Cyber Intelligence and Information Security \\ Sapienza University of Rome
}

\author{
A. Margheri and V. Sassone \\ University of Southampton
}

\begin{abstract}
As data is having an increasingly relevant role in different business fields, ensuring integrity has become fundamental. Modern databases rely on transaction history written on redo logs to allow for data restore. However, if redo logs are (maliciously) forged, data can actually be lost or altered. Due its strong data integrity guarantees, blockchain technology can be employed to ensure log integrity, but its current performance limitations hinder actual exploitations.

In previous work, we proposed a layered blockchain-based architecture for distributed (federated) database redo logs: a fast first layer blockchain, anchored to a secure second layer blockchain, based on proof-of-work to achieve strong integrity. Here, we present an implementation and an experimental evaluation of a prototype of that architecture, which employs a total consensus algorithm on the first layer blockchain. Finally, to improve availability and scalability, we refine our solution by investigating, respectively, a Byzantine Fault Tolerant consensus and a Distributed Hash Table solution to shard the first layer blockchain ledger among available nodes.
\end{abstract}

\section{Introduction}

As a critical component of many systems, data has an appealing target for cyber-attacks. Tampering with data can go undetected and drive malicious operations, e.g. data alteration and deletion. Most of all, differently from availability loss, data integrity can be hardly restored once lost. Modern database systems use logging mechanisms to track data changes, e.g. Write-Ahead Logging of PostgreSQL ${ }^{1}$ and Redo Log of Oracle 2 . However, if such logging files are forged, recognising an attack or a failure is awkward as data integrity relies too intimately on the system itself. Typically, Remote Data Auditing mitigations are employed, but they come with high costs and rely on trusted third parties [10].

In recent years blockchain came to prominence through the Bitcoin system [7], and have emerged since as a powerful technology to provide strong data integrity guarantees in trust-less networks. Blockchain ensures integrity by means of proof-of-work (PoW), a consensus schema based on

1. postgresql.org/docs/9.1/static/wal-intro.html

2. docs.oracle.com/cd/B19306_01/server.102/b14231/onlineredo.htm solving a crypto-puzzles that is considered computationally inviolable [4]. Blockchain also offers so-called smart contracts, immutable programs deployed and executed on top of a blockchain system, e.g. Ethereum [11], to realise decentralised applications where no involved party is in control neither of the code nor of the data.

Regardless of strong integrity guarantees, exploiting blockchain to strengthen (distributed) database systems implies coping with overwhelming performance penalties: viz., high latency and low throughput. Indeed, preliminary blockchain-based database solutions rationalise blockchain features: BigchainDB [6] replaces PoW (and its security) with a lightweight protocol; RSCoin [2] (re)introduces certain degree of centralisation in the system.

In [3] we proposed a blockchain-based architecture for distributed (federated) database transaction (or redo) logs, which permits achieving both high performance and adequate integrity guarantees. Specifically, we defined a layered architecture that makes use of a first layer blockchain assuring low latency and high throughput, anchored to a second layer blockchain featuring PoW to ensure data integrity.

In this paper, we present a prototype implementation of that architecture, which features a total consensus algorithm on the first layer blockchain, and its experimental evaluation. Then, to cope with faults and Denial-of-Service (DoS) attacks, we investigate a Byzantine Fault Tolerant consensus. Finally, to improve scalability, we investigate a Distributed Hash Table (DHT) as storage means for the first layer.

As a case study, we address the distributed database underlying FaaS [8], a recent Cloud Federation solution put forward by the EU H2020 SUNFISH project. FaaS offers a democratic federation of Clouds which crucially relies on a distributed database to ensure that no federation member can tamper with federated data. Clearly, the requirements of the application demand not only data integrity, but also adequate performance. The deployment of a fast and secure blockchain-based system is indeed paramount to achieve both performance and democracy requirements of FaaS.

Paper structure. $\$ 2$ introduces the layered architecture; $\$ 3$ presents and evaluates the prototype. $\$ 4$ illustrates the proposed extensions. $\$ 5$ concludes and discusses future work. 


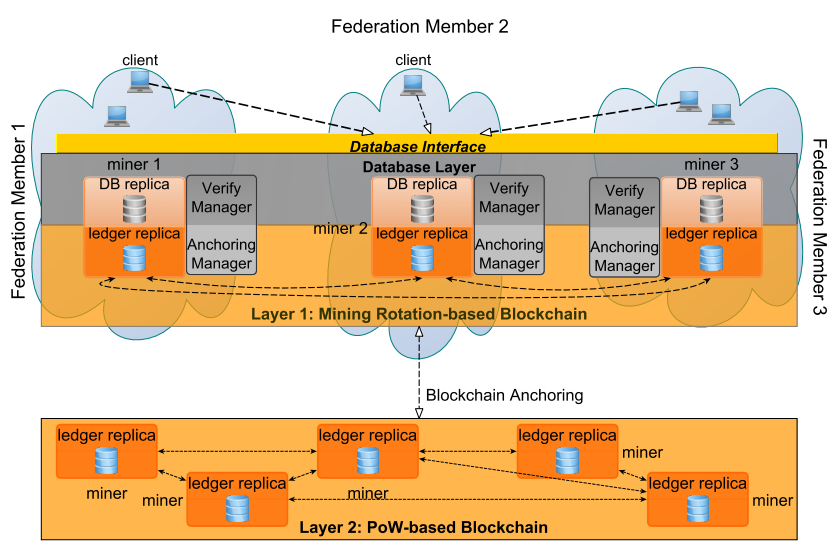

Figure 1. 2LBC architecture for a FaaS federation

\section{Background}

In [3] we posed some important open research questions on the issues of employing blockchain "as-is" in database settings, and pointed out practical research directions towards its effective employment: improving performance while ensuring data integrity. To this aim, we proposed a 2layer blockchain architecture (2LBC), enabling a replicated, trustworthy redo log for a distributed (federated) database.

2LBC consists of a permissioned blockchain on the first layer and a public permissionless blockchain on the second layer. The first layer features a fast consensus algorithm based on a leader rotation approach: the time is split into rounds each of which has a designed leader (i.e., a miner) chosen according to a deterministic fair policy. The leader defines the ordering of transactions (txns) containing the operations tracked in the redo log (aka ledger). Transactions are stored, once signed with asymmetric cryptography by all members, in each ledger replica.

Figure 11 shows the 2LBC architecture in the context of a FaaS federation. Specifically, each federation member contributes to $2 \mathrm{LBC}$ with one miner-distinguished network nodes building the integrity of blockchain- keeping both database and ledger replicas. The second layer features PoW and is used to guarantee the integrity of the first layer. Periodically, the hash of the first layer blockchain is here stored via the Anchoring Manager. PoW ensures the immutability of such hashes. These hashes, so, act as forensic evidences of the first layer: if a malicious miner maliciously tries to alter the redo $\log$, the first layer blockchain will result forged as the hash stored in the second layer will be different.

\section{Prototype Implementation and Evaluation}

We developed the first layer blockchain in Java. Miners join a $\mathrm{p} 2 \mathrm{p}$ network and communicate through txns composed by a payload (i.e., client operation, a sequence number and a timestamp) and a certificate with miners' signatures. Miners trade messages either directly through JavaRMI or in broadcast through JGroup (http://jgroups.org/). Clients can operate on the database by issuing to all miners, through JavaRMI, two kind of operations: (i) $\operatorname{set}(\mathrm{k}, \mathrm{v})$ to assign a value $\mathrm{v}$ to a key $\mathrm{k}$ (it returns to the client a boolean confirmation); (ii) get (k) to obtain the value stored to key $\mathrm{k}$ by returning the txn related to the last set toward $\mathrm{k}$.

Consensus. In the case of set, miners have to achieve consensus to give confirmation to the client. The consensus is based on a three-phase commit protocol [9]. Practically, a client broadcasts a set $_{i}$ operation to all miners which verify its correctness through a Verify Manager and add it to a queue. Once the current leader (who is in charge of ordering txns) proposes $\operatorname{txn}_{i}$ related to $\mathrm{set}_{i}$, other miners remove it from their queue and broadcast their signatures to build the corresponding certificate. When the certificate contains all the signatures, all miners commit the txn in their ledger replica and trigger the update in the database replica.

In the case of get, every miner answers to the client with the last $\operatorname{set}(\mathrm{k}, \mathrm{v})$ stored txn for the key $\mathrm{k}$, so the client can obtain the value $\mathrm{v}$ from the received txn having the more recent timestamp and verify the correctness with the miners' signatures.

Anchoring. When the round time of a leader terminates, it triggers a leader change, which amounts to stores a special txn, and the anchoring procedure. Specifically, the abdicating leader computes the SHA-1 hash of the first layer blockchain and sends a witness txn to a dedicated witness smart-contract on the second layer blockchain 3

Evaluation. We evaluated our prototype on a private cluster composed by $N=6$ Ubuntu 16.04 Virtual Machines (VMs) each one running a miner process, deployed on 4 blade servers IBM HS22, equipped with 2 Quad-Core Intel Xeon X5560 2.28 GHz CPUs and 24 GB RAM.

Network latency between miners is simulated according to a Poisson distribution in the range 5-20 ms. Operations set and get on random keys are injected with different rates via 2 multi-thread clients deployed on 2 further VMs. To evaluate $2 \mathrm{LBC}$ performances, we measured throughput and response time of the operations over time.

For set operations, Figure 2(a) (resp., 2(b) reports throughput (resp., latency) results that, as expected, are much higher (resp., lower) than Ethereum. Above 240 Op/s, they become unstable for resource saturation due to high number of messages exchanged; indeed, the total consensus algorithm needs that miners trade all their signatures for each operation. Additionally, leader rotation introduces an overhead on the enqueued operations during leader changes; we refer to transient state as the transitory period where latency exceeds $50 \%$ of the average latency. As shown in Figure 3, increasing the round time makes the transient state shorter. On the contrary, frequent leader changes increase such overhead.

For get operations, Figures 2(c) and 2(d) report throughput and latency results. Notably, as there is no transient state for get (i.e., miners just send their last local value regardless leader changes), the results are comparable both with and without leader rotation.

3. The smart contract is implemented for Ethereum. 


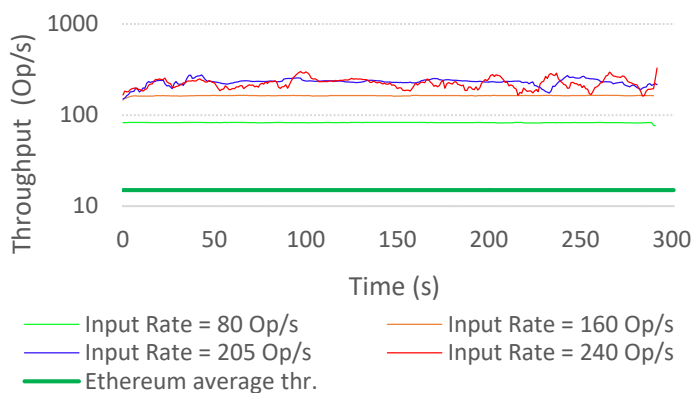

(a) Throughput of set operations for different input rates

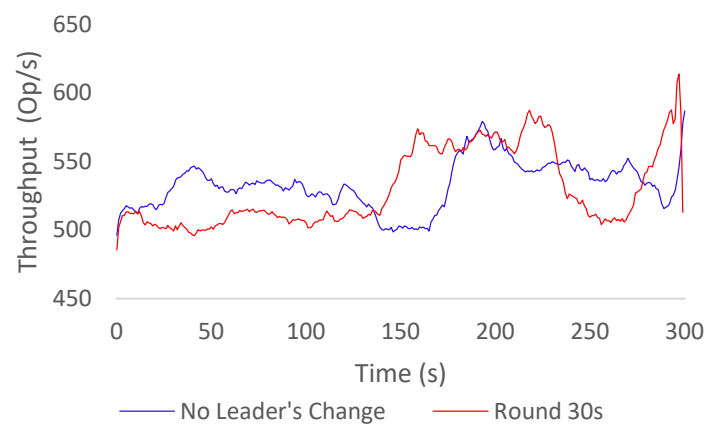

(c) Throughput for get operations while injecting $\approx 500 \mathrm{op} / \mathrm{s}$ without changing leader and with 30 s leader round time

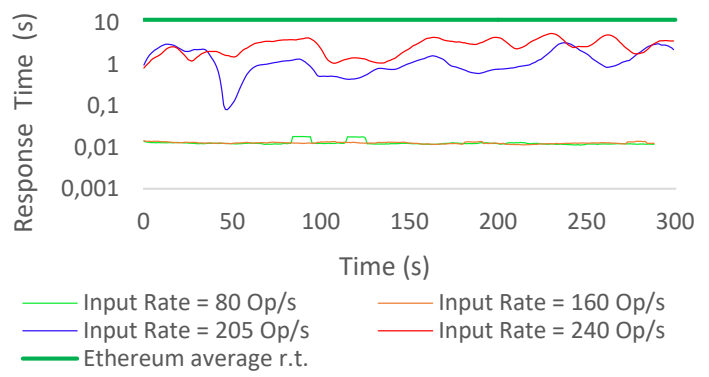

(b) Response Time of set operations for different input rates

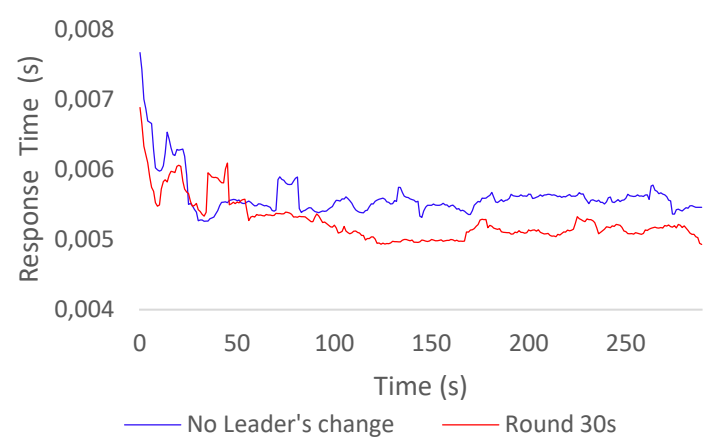

(d) Response Time for get operations while injecting $\approx 500$ op/s without changing leader and with 30 s leader round time

Figure 2. Throughput and Response Time evaluation of 2LBC for set and get operations

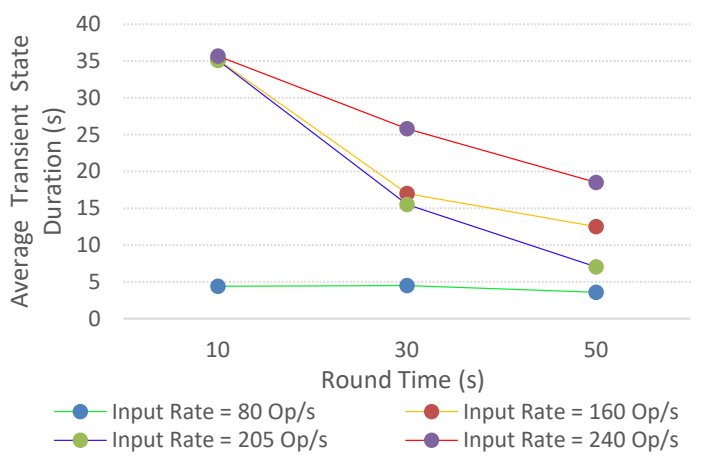

Figure 3. Analysis of the relation between round time (x axis) and transient state duration (y axis) for different input rates

Security Analysis. We modelled three possible kinds of attack: A1 tampering, if miner tries to modify the log; $A 2$ forgering, if miner tries to send a fake txn, i.e. by forging a client operation; $A 3 D o S$, either a miner does not sign a txn or a leader does not broadcast a txn.

In our approach a valid txn must contain a certificate with all miners' signatures. This avoids miners from maliciously update values without informing other members $(A 1)$, and from creating fake txns (A2) (unless it can obtain the private keys of all other miners). Moreover, hashes stored in the second layer blockchain are immutable, hence, though the attacker is able to compromise the first layer by stealing all miners' keys, $A 2$ might be detected by comparing the hash of the first layer with the hash evidence stored on the second layer; to compromise also hashes in the second layer the attacker should obtain a significant, unfeasible computational power [4]. Furthermore, the leader cannot forward fake txns (A2), because miners sign only txns related to operations that they enqueued. Specifically, miners verify the correctness of txn fields being sure that are not forged.

Our approach is instead vulnerable to DoS attacks $(A 3)$ : a single malicious miner can block set operations not sending its signature or a malicious leader (during its leading periods) can avoid to forward a txn. This cannot happen with get operations, as miners return their response independently. Indeed, if there is at least one honest miner, a client can obtain the value by its corresponding txn and verify the signatures in the certificate to prove the authenticity.

\section{Improving Availability and Scalability}

The main limitations of the current $2 \mathrm{LBC}$ prototype are related to availability issues (as explained in last section) and scalability. Indeed, overall system performance does not scale adding new nodes, as the used total consensus algorithm has lower performance with additional nodes. In this section we propose the solutions to cope with them.

Availability. To mitigate such limitations, we propose a Byzantine Fault Tolerant solution based on PBFT [1]. To tolerate up to $f$ Byzantine miners it requires $3 f+1$ miners to provide both safety and liveness. This leads to higher 


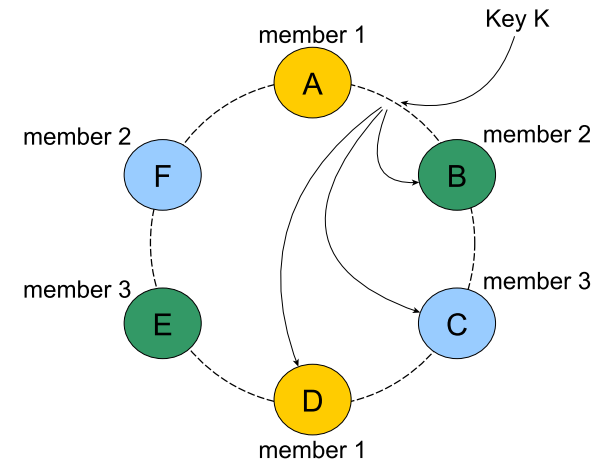

Figure 4. Example of the DHT-based ledger solution to achieve total replication in the SUNFISH scenario. The federation is composed by $M=3$ members (each one marked with a proper color), each one exposing $N=2$ miners (identified by a proper letter). Miners are disposed on the ring so as each member has all keys of the database sharded between its miners. The single miners keep only a subset proportional to the replication factor. In the example, the replication factor is $M=3$ and each miner maintains only $1 / N=1 / 2$ of database keys.

availability level as honest miners need to wait just $f+1$ valid signatures to commit a txn. We can so tolerate up to $f$ silent miners at the cost of weaker integrity guarantees; indeed data corruption is possible with just $f+1$ compromised miners, rather than all $N$ when total consensus is used. Anyway, the integrity of txns already witnessed in the second layer is still ensured thanks to the PoW.

Scalability. To improve scalability, we propose a data sharding solution for permissioned (federated) blockchain, similar to [5] for permissionless blockchain. Specifically, we introduce a DHT-based ledger in which each miner, on the base of a keyspace partitioning, only handles txns for specific subsets of keys. This approach permits tuning txn loads on miners and, consequently, makes the system more scalable. Furthermore, each key range has a configurable replication factor to enable fault tolerance. In our solution, contrarily to common DHT implementations, the miners involved in a set operation must achieve consensus before writing the operation in the local replica (hence in the local keyspace) of the redo log. This permits achieving strong consistency, hence avoiding consistency issues which mar well known DHT-based NoSQL databases, such as DynamoDB ${ }^{4}$ and Cassandr 55 Clearly, this comes at the cost of performance penalties, whose quantification depends on the technology and is beyond our scope here.

The solution to the SUNFISH scenario can e.g. change as follow: let $M$ be the number of federation members, each providing $N$ (rather than a single) miners. The DHT ring includes $M \cdot N$ nodes, the replication factor is set to $M$, and miners can be placed over the ring so that the miners of each federation member collectively manage all the keys. Figure 4 shows an example of a SUNFISH federation composed by $M=3$ members, each exposing $N=2$ miners.

\footnotetext{
4. aws.amazon.com/dynamodb/faqs/

5. wiki.apache.org/cassandra/ArchitectureOverview
}

\section{Conclusion}

We evaluated a prototype of $2 \mathrm{LBC}$, a 2-layered architecture for a blockchain-based database able to provide both high performance and strong data integrity guarantees in a totally decentralised environment. We proposed also a solution to cope with current availability issues and a DHTbased ledger to shard data and make the system scalable.

In the future we will continue developing and evaluating our prototype by introducing the solutions based on PBFT and DHT. In order to compare alternative solutions, we plan to develop a metric for data integrity based on the effort required for an attacker to tamper with data without being noticed. This metric will allow us to define and evaluate varying tradeoff levels between system availability, integrity and performance with a different number of miners participating the consensus protocol. Finally, we aim to evaluate a scenario where each member can expose a different number of miners.

\section{Acknowledgment}

This work has been supported by the EU H2020 SUNFISH project, grant N.644666.

\section{References}

[1] Miguel Castro, Barbara Liskov, et al. Practical byzantine fault tolerance. In OSDI, volume 99, pages 173-186, 1999.

[2] George Danezis and Sarah Meiklejohn. Centrally Banked Cryptocurrencies. In 23rd Annual Network and Distributed System Security Symposium, NDSS, 2016.

[3] Edoardo Gaetani, Leonardo Aniello, Roberto Baldoni, Federico Lombardi, Andrea Margheri, and Vladimiro Sassone. Blockchain-based database to ensure data integrity in cloud computing environments. ITA-SEC. CEUR-WS.org, 2017.

[4] Juan Garay, Aggelos Kiayias, and Nikos Leonardos. The Bitcoin Backbone Protocol: Analysis and Applications, pages 281-310. Springer Berlin Heidelberg, Berlin, Heidelberg, 2015.

[5] Loi Luu, Viswesh Narayanan, Chaodong Zheng, Kunal Baweja, Seth Gilbert, and Prateek Saxena. A secure sharding protocol for open blockchains. In Proceedings of the 2016 ACM SIGSAC Conference on Computer and Communications Security, pages 17-30. ACM, 2016.

[6] Trent McConaghy, Rodolphe Marques, Andreas Müller, Dimitri De Jonghe, Troy McConaghy, Greg McMullen, Ryan Henderson, Sylvain Bellemare, and Alberto Granzotto. BigchainDB: A Scalable Blockchain Database. 2016.

[7] Satoshi Nakamoto. Bitcoin: A peer-to-peer electronic cash system, 2008. Available at https://bitcoin.org/bitcoin.pdf

[8] Francesco Paolo Schiavo, Vladimiro Sassone, Luca Nicoletti, and Andrea Margheri (Eds.). FaaS: Federation-as-a-Service, 2016. Technical Report. Available at https://arxiv.org/abs/1612.03937

[9] Dale Skeen and Michael Stonebraker. A formal model of crash recovery in a distributed system. IEEE Transactions on Software Engineering, (3):219-228, 1983.

[10] Mehdi Sookhak, Abdullah Gani, Hamid Talebian, Adnan Akhunzada, Samee U. Khan, Rajkumar Buyya, and Albert Y. Zomaya. Remote Data Auditing in Cloud Computing Environments: A Survey, Taxonomy, and Open Issues. ACM Comput. Surv., 47(4):65:1-65:34, 2015.

[11] Gavin Wood. Ethereum: A secure decentralised generalised transaction ledger. Ethereum Project Yellow Paper, 2014. 